Supporting Information for

\title{
Synthesis, Structure, and Characterization of Novel
}

\section{Two- and Three-Dimensional Vanadates:}

\section{$\mathrm{Ba}_{2.5}\left(\mathrm{VO}_{2}\right)_{3}\left(\mathrm{SeO}_{3}\right)_{4} \cdot \mathrm{H}_{2} \mathrm{O}$ and $\mathrm{La}\left(\mathrm{VO}_{2}\right)_{3}\left(\mathrm{TeO}_{6}\right) \cdot 3 \mathrm{H}_{2} \mathrm{O}$}

T. Sivakumar, Kang Min Ok, and P. Shiv Halasyamani*

Department of Chemistry and Center for Materials Chemistry, University of Houston, 136 Fleming Building, Houston, Texas 77204-5003

Figure S1. ORTEP representation $(50 \%$ probability ellipsoids $)$ of $\mathrm{Ba}_{2.5}\left(\mathrm{VO}_{2}\right)_{3}\left(\mathrm{SeO}_{3}\right)_{4} \cdot \mathrm{H}_{2} \mathrm{O}$

Figure S2. ORTEP representation (50\% probability ellipsoids) of $\mathrm{La}\left(\mathrm{VO}_{2}\right)_{3}\left(\mathrm{TeO}_{6}\right) \cdot 3 \mathrm{H}_{2} \mathrm{O}$

Figure S3. ORTEP representation of 12- and 8-membered rings in $\mathrm{Ba}_{2.5}\left(\mathrm{VO}_{2}\right)_{3}\left(\mathrm{SeO}_{3}\right)_{4} \cdot \mathrm{H}_{2} \mathrm{O} .50 \%$ probability ellipsoids ( $\mathrm{O}$ in red, $\mathrm{V}$ in blue, and $\mathrm{Se}$ in green) are depicted. Note the 12-membered rings are in eclipsed conformation.

Figure S4. ORTEP representation of the $\mathrm{TeO}_{6}$ and $\mathrm{VO}_{4}$ polyhedra in $\mathrm{La}\left(\mathrm{VO}_{2}\right)_{3}\left(\mathrm{TeO}_{6}\right) \cdot 3 \mathrm{H}_{2} \mathrm{O} .50 \%$ probability ellipsoids $(\mathrm{O}$ in red, $\mathrm{V}$ in blue, and $\mathrm{Te}$ in green) are depicted. Note that each $\mathrm{TeO}_{6}$ polyhedron is surrounded by six $\mathrm{VO}_{4}$ tetrahedra.

Figure S5. Calculated (a) and observed (b) powder X-ray diffraction (XRD) patterns for $\mathrm{Ba}_{2.5}\left(\mathrm{VO}_{2}\right)_{3}\left(\mathrm{SeO}_{3}\right)_{4} \cdot \mathrm{H}_{2} \mathrm{O}$. XRD pattern for anhydrous $\mathrm{Ba}_{2.5}\left(\mathrm{VO}_{2}\right)_{3}\left(\mathrm{SeO}_{3}\right)_{4}$, obtained by dehydration of $\mathrm{Ba}_{2.5}\left(\mathrm{VO}_{2}\right)_{3}\left(\mathrm{SeO}_{3}\right)_{4} \cdot \mathrm{H}_{2} \mathrm{O}$ at $340{ }^{\circ} \mathrm{C}$ for $1 \mathrm{~h}$, is shown in the top panel (c). 
Figure S6. Powder X-ray diffraction patterns for (a) $\mathrm{La}\left(\mathrm{VO}_{2}\right)_{3}\left(\mathrm{TeO}_{6}\right) \cdot 3 \mathrm{H}_{2} \mathrm{O}$ (calculated) (b) $\mathrm{La}\left(\mathrm{VO}_{2}\right)_{3}\left(\mathrm{TeO}_{6}\right) \cdot 3 \mathrm{H}_{2} \mathrm{O}$ (observed) (c) anhydrous $\mathrm{La}\left(\mathrm{VO}_{2}\right)_{3}\left(\mathrm{TeO}_{6}\right)$, obtained by dehydration of $\mathrm{La}\left(\mathrm{VO}_{2}\right)_{3}\left(\mathrm{TeO}_{6}\right) \cdot 3 \mathrm{H}_{2} \mathrm{O}$ at $350{ }^{\circ} \mathrm{C}$, and (d) products obtained by calcining $\mathrm{La}\left(\mathrm{VO}_{2}\right)_{3}\left(\mathrm{TeO}_{6}\right) \cdot 3 \mathrm{H}_{2} \mathrm{O}$ at $600{ }^{\circ} \mathrm{C}$ showing $\mathrm{LaVO}_{4}$ is the major phase.

Table S1. Powder XRD data (indexing using PROSZKI program) for anhydrous $\mathrm{La}\left(\mathrm{VO}_{2}\right)_{3}\left(\mathrm{TeO}_{6}\right)$

Figure S7. IR spectra for $\mathrm{Ba}_{2.5}\left(\mathrm{VO}_{2}\right)_{3}\left(\mathrm{SeO}_{3}\right)_{4} \cdot \mathrm{H}_{2} \mathrm{O}$

Figure S8. IR spectra for $\mathrm{La}\left(\mathrm{VO}_{2}\right)_{3}\left(\mathrm{TeO}_{6}\right) \cdot 3 \mathrm{H}_{2} \mathrm{O}$

Figure S9. Raman spectra for (a) $\mathrm{Ba}_{2.5}\left(\mathrm{VO}_{2}\right)_{3}\left(\mathrm{SeO}_{3}\right)_{4} \cdot \mathrm{H}_{2} \mathrm{O}$, and (b) $\mathrm{La}\left(\mathrm{VO}_{2}\right)_{3}\left(\mathrm{TeO}_{6}\right) \cdot 3 \mathrm{H}_{2} \mathrm{O}$

Figure S10. Diffuse reflectance spectra for $\mathrm{Ba}_{2.5}\left(\mathrm{VO}_{2}\right)_{3}\left(\mathrm{SeO}_{3}\right)_{4} \cdot \mathrm{H}_{2} \mathrm{O}$, and $\mathrm{La}\left(\mathrm{VO}_{2}\right)_{3}\left(\mathrm{TeO}_{6}\right) \cdot 3 \mathrm{H}_{2} \mathrm{O}$

Figure S11. Thermogravimetric analysis curve for $\mathrm{Ba}_{2.5}\left(\mathrm{VO}_{2}\right)_{3}\left(\mathrm{SeO}_{3}\right)_{4} \cdot \mathrm{H}_{2} \mathrm{O}$

Figure S12. Nitrogen Adsorption Isotherm and BET plot for $\mathrm{Ba}_{2.5}\left(\mathrm{VO}_{2}\right)_{3}\left(\mathrm{SeO}_{3}\right)_{4}$.

Figure S13. Thermogravimetric analysis curve for $\mathrm{La}\left(\mathrm{VO}_{2}\right)_{3}\left(\mathrm{TeO}_{6}\right) \cdot 3 \mathrm{H}_{2} \mathrm{O}$ 

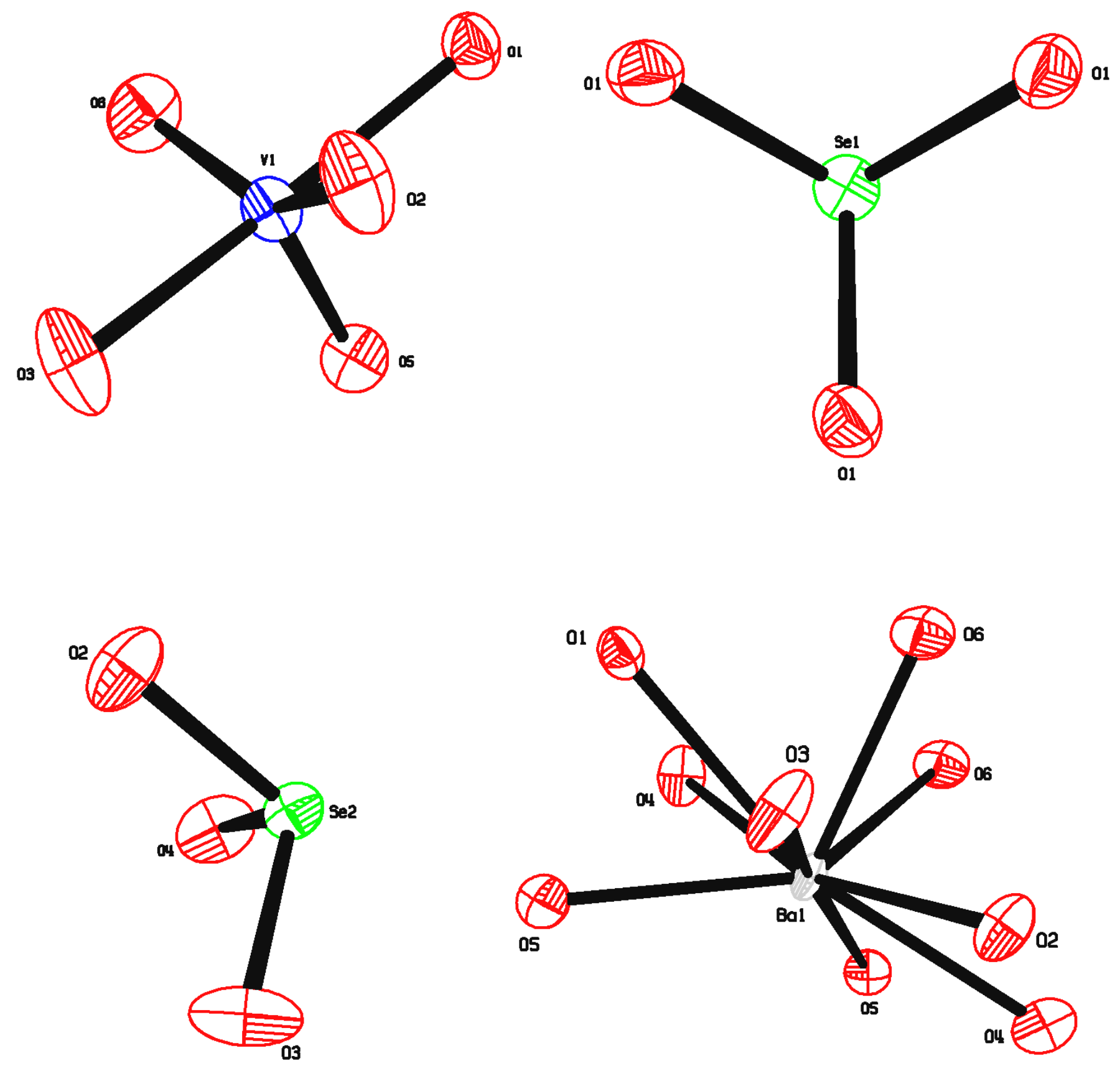

Figure S1. ORTEP representation $(50 \%$ probability ellipsoids $)$ of $\mathrm{Ba}_{2.5}\left(\mathrm{VO}_{2}\right)_{3}\left(\mathrm{SeO}_{3}\right)_{4} \cdot \mathrm{H}_{2} \mathrm{O}$ 

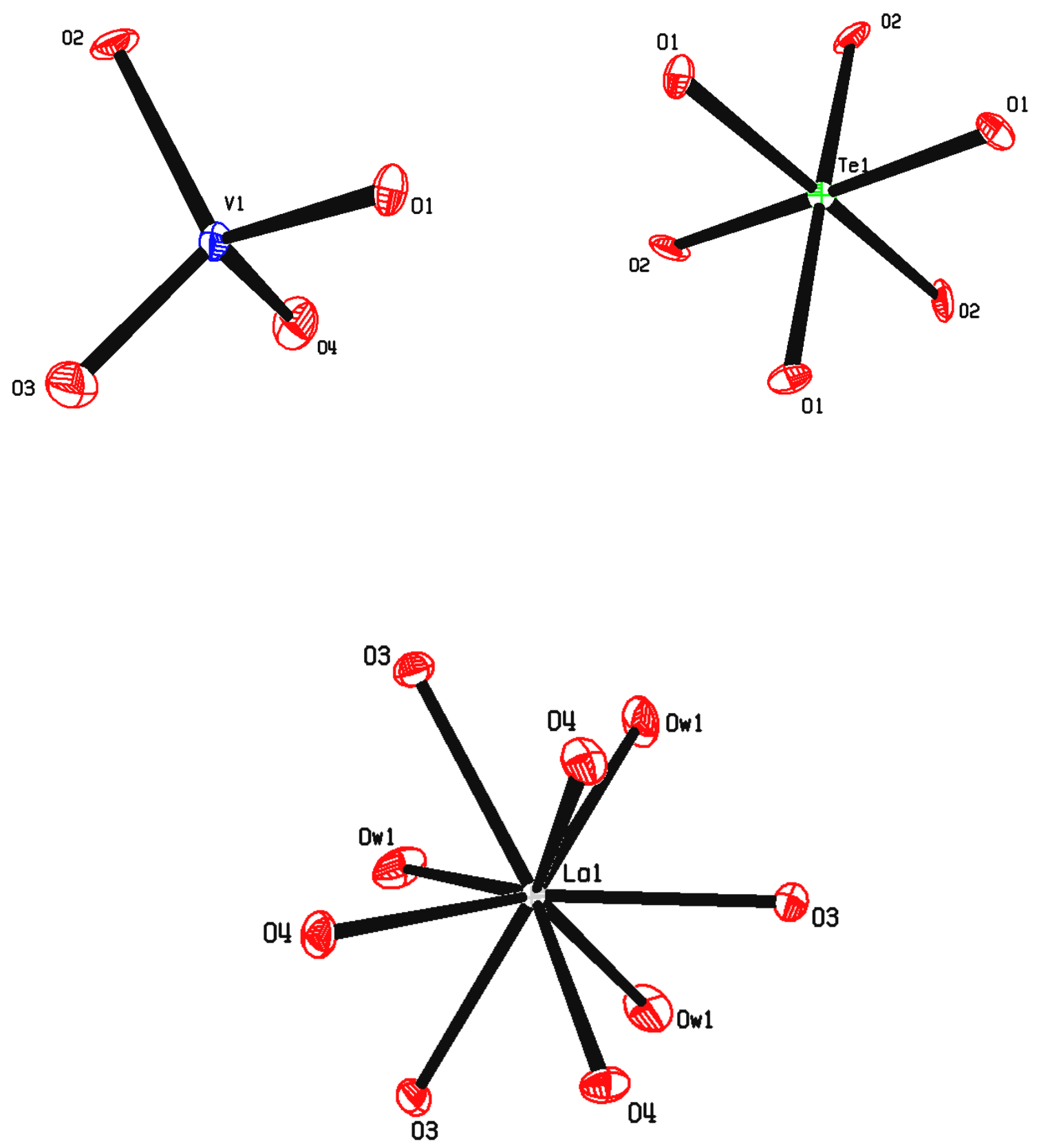

Figure S2. ORTEP representation (50\% probability ellipsoids) of $\mathrm{La}\left(\mathrm{VO}_{2}\right)_{3}\left(\mathrm{TeO}_{6}\right) \cdot 3 \mathrm{H}_{2} \mathrm{O}$ 


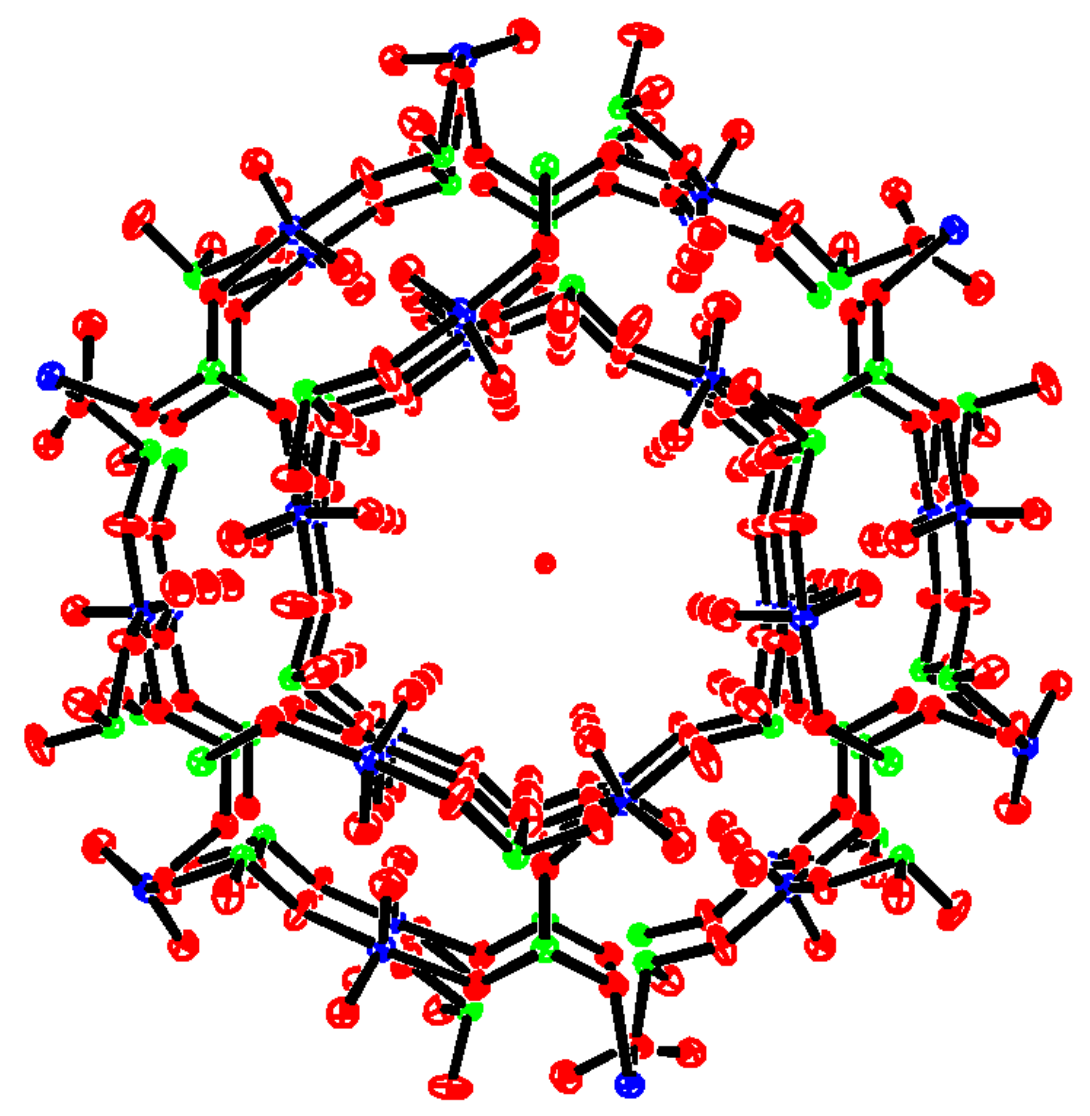

Figure S3. ORTEP representation of 12- and 8-membered rings in $\mathrm{Ba}_{2.5}\left(\mathrm{VO}_{2}\right)_{3}\left(\mathrm{SeO}_{3}\right)_{4} \cdot \mathrm{H}_{2} \mathrm{O} .50 \%$ probability ellipsoids ( $\mathrm{O}$ in red, $\mathrm{V}$ in blue, and $\mathrm{Se}$ in green) are depicted. Note the 12-membered rings are in eclipsed conformation. 


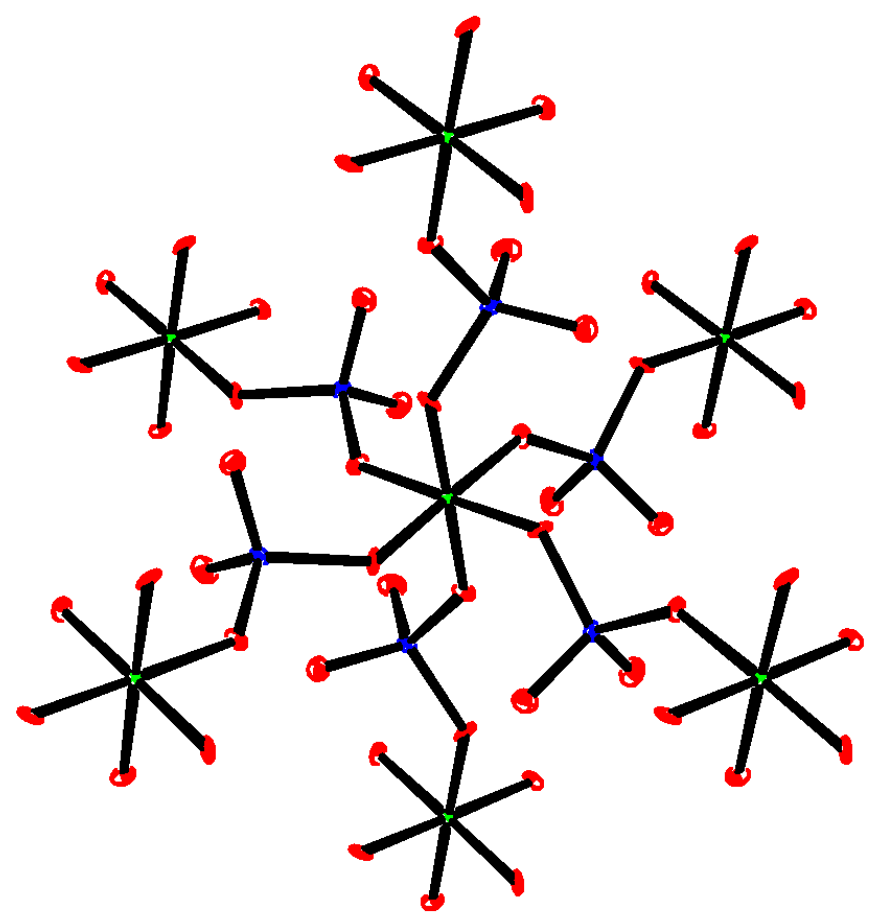

Figure S4. ORTEP representation of the $\mathrm{TeO}_{6}$ and $\mathrm{VO}_{4}$ polyhedra in $\mathrm{La}\left(\mathrm{VO}_{2}\right)_{3}\left(\mathrm{TeO}_{6}\right) \cdot 3 \mathrm{H}_{2} \mathrm{O} .50 \%$ probability ellipsoids $(\mathrm{O}$ in red, $\mathrm{V}$ in blue, and $\mathrm{Te}$ in green $)$ are depicted. Note that each $\mathrm{TeO}_{6}$ polyhedron is surrounded by six $\mathrm{VO}_{4}$ tetrahedra. 


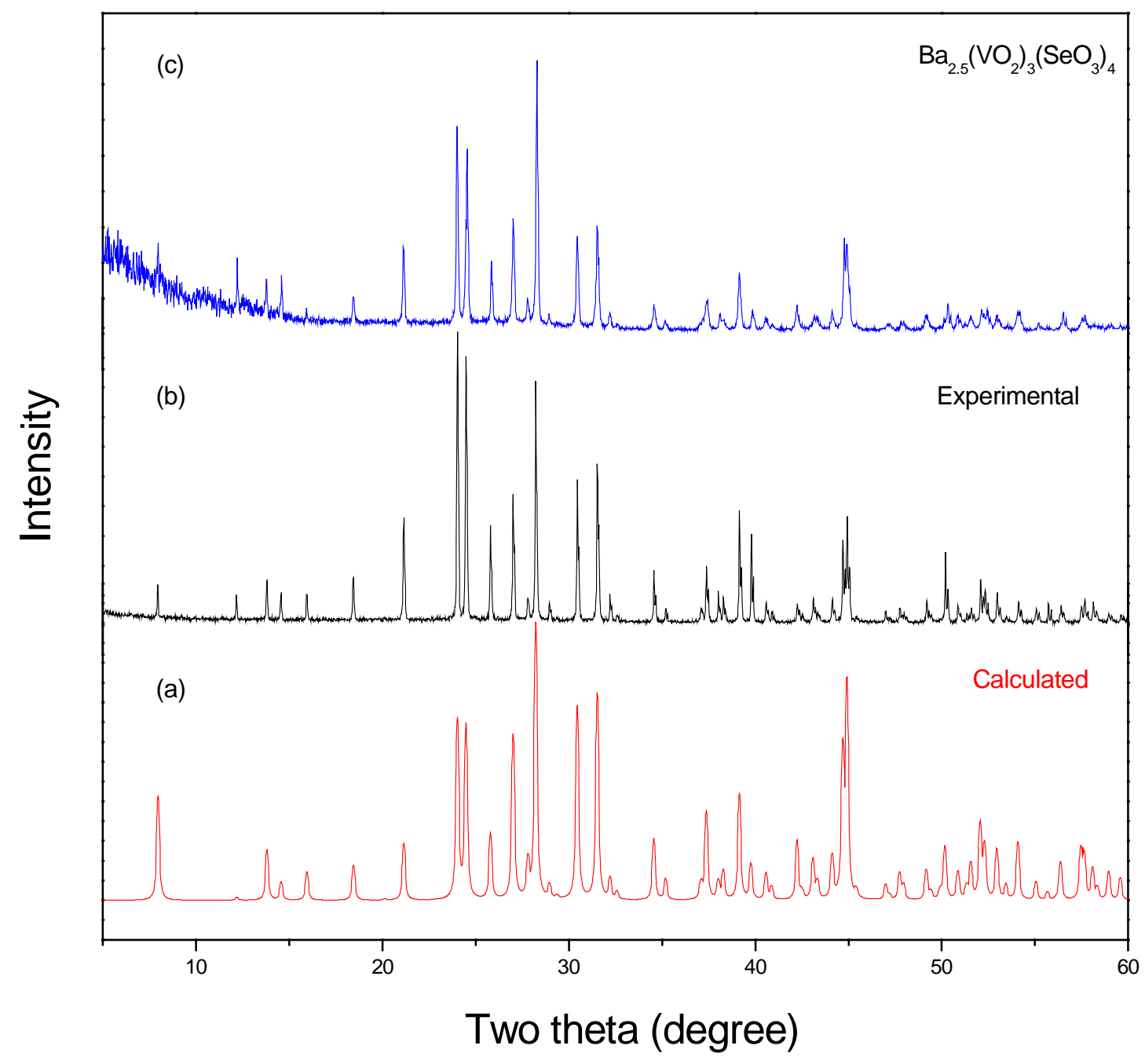

Figure S5. Calculated (a) and observed (b) powder X-ray diffraction (XRD) patterns for $\mathrm{Ba}_{2.5}\left(\mathrm{VO}_{2}\right)_{3}\left(\mathrm{SeO}_{3}\right)_{4} \cdot \mathrm{H}_{2} \mathrm{O}$. XRD pattern for anhydrous $\mathrm{Ba}_{2.5}\left(\mathrm{VO}_{2}\right)_{3}\left(\mathrm{SeO}_{3}\right)_{4}$, obtained by dehydration of $\mathrm{Ba}_{2.5}\left(\mathrm{VO}_{2}\right)_{3}\left(\mathrm{SeO}_{3}\right)_{4} \cdot \mathrm{H}_{2} \mathrm{O}$ at $340{ }^{\circ} \mathrm{C}$ for $1 \mathrm{~h}$, is shown in the top panel (c). 


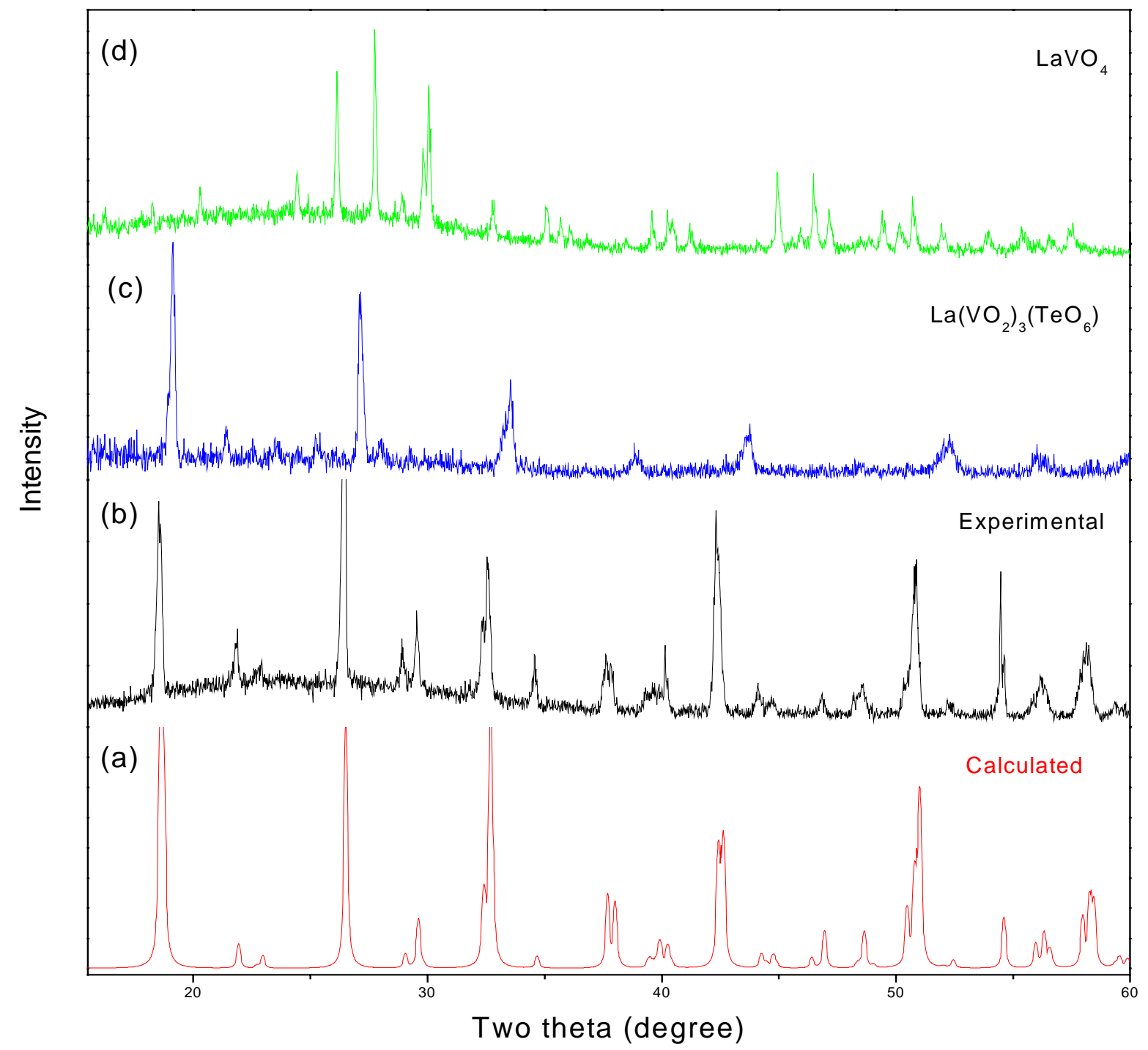

Figure S6. Powder X-ray diffraction patterns for (a) $\mathrm{La}\left(\mathrm{VO}_{2}\right)_{3}\left(\mathrm{TeO}_{6}\right) \cdot 3 \mathrm{H}_{2} \mathrm{O}$ (calculated) (b) $\mathrm{La}\left(\mathrm{VO}_{2}\right)_{3}\left(\mathrm{TeO}_{6}\right) \cdot 3 \mathrm{H}_{2} \mathrm{O}$ (observed) (c) anhydrous $\mathrm{La}\left(\mathrm{VO}_{2}\right)_{3}\left(\mathrm{TeO}_{6}\right)$, obtained by dehydration of $\mathrm{La}\left(\mathrm{VO}_{2}\right)_{3}\left(\mathrm{TeO}_{6}\right) \cdot 3 \mathrm{H}_{2} \mathrm{O}$ at $350{ }^{\circ} \mathrm{C}$, and (d) products obtained by calcining $\mathrm{La}\left(\mathrm{VO}_{2}\right)_{3}\left(\mathrm{TeO}_{6}\right) \cdot 3 \mathrm{H}_{2} \mathrm{O}$ at $600{ }^{\circ} \mathrm{C}$ showing $\mathrm{LaVO}_{4}{ }^{1}$ is the major phase.

${ }^{1}$ JCPDS card no. 70-0216 
Table S1. Powder XRD data (indexing using PROSZKI ${ }^{1}$ program) for anhydrous $\mathrm{La}\left(\mathrm{VO}_{2}\right)_{3}\left(\mathrm{TeO}_{6}\right)$

\begin{tabular}{llllll}
\hline $\mathrm{h}$ & $\mathrm{k}$ & $\mathrm{l}$ & $\mathrm{d}_{\mathrm{obs}}$ & $\mathrm{d}^{*}$ cal & $\mathrm{I}_{\mathrm{obs}}$ \\
\hline 1 & 0 & 4 & 4.642 & 4.644 & 100 \\
1 & 1 & 0 & 4.623 & 4.628 & 85 \\
2 & 0 & 2 & 3.773 & 3.781 & 12 \\
0 & 2 & 4 & 3.282 & 3.278 & 79 \\
0 & 1 & 8 & 2.686 & 2.684 & 26 \\
2 & 1 & 4 & 2.668 & 2.675 & 35 \\
2 & 0 & 8 & 2.320 & 2.322 & 14 \\
2 & 1 & 8 & 2.077 & 2.075 & 16 \\
1 & 3 & 4 & 2.070 & 2.071 & 24 \\
1 & 1 & 12 & 1.757 & 1.757 & 16 \\
4 & 1 & 0 & 1.751 & 1.749 & 19 \\
0 & 4 & 8 & 1.639 & 1.639 & 14
\end{tabular}

*Space group $R 3 c ; a=b=9.256(5) \AA, c=22.79(2) \AA$ and $\mathrm{V}=1691(2) \AA^{3}$.

${ }^{1}$ Losocha, W.; Lewinski, K. J. Appl. Crystallogr. 1994, 27, 437-438. 


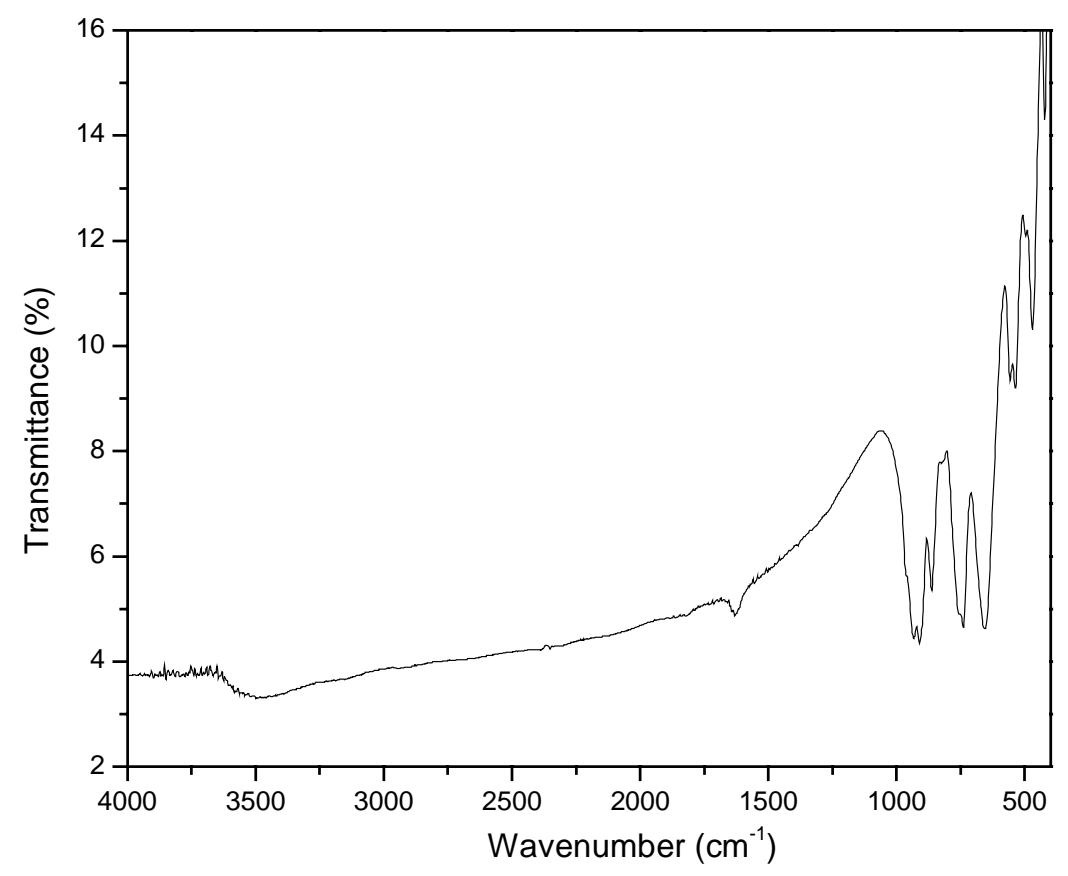

Figure S7. IR spectra for $\mathrm{Ba}_{2.5}\left(\mathrm{VO}_{2}\right)_{3}\left(\mathrm{SeO}_{3}\right)_{4} \cdot \mathrm{H}_{2} \mathrm{O}$

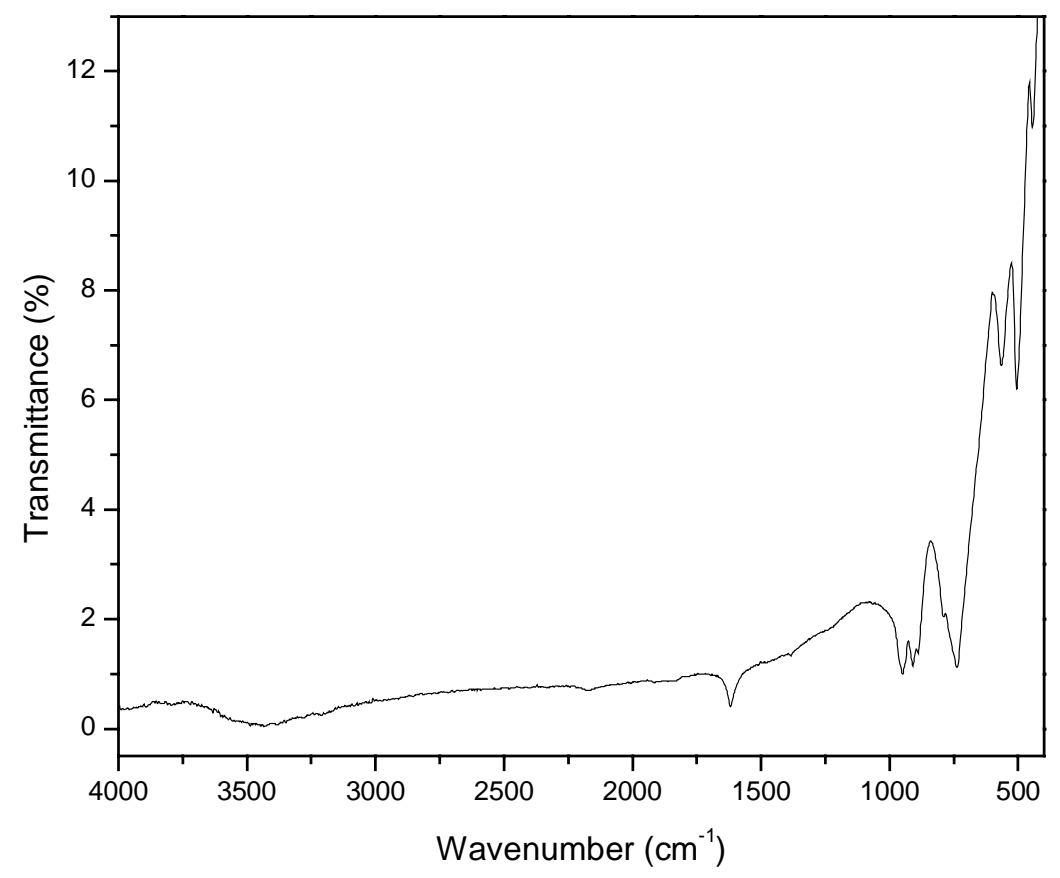

Figure S8. IR spectra for $\mathrm{La}\left(\mathrm{VO}_{2}\right)_{3}\left(\mathrm{TeO}_{6}\right) \cdot 3 \mathrm{H}_{2} \mathrm{O}$ 

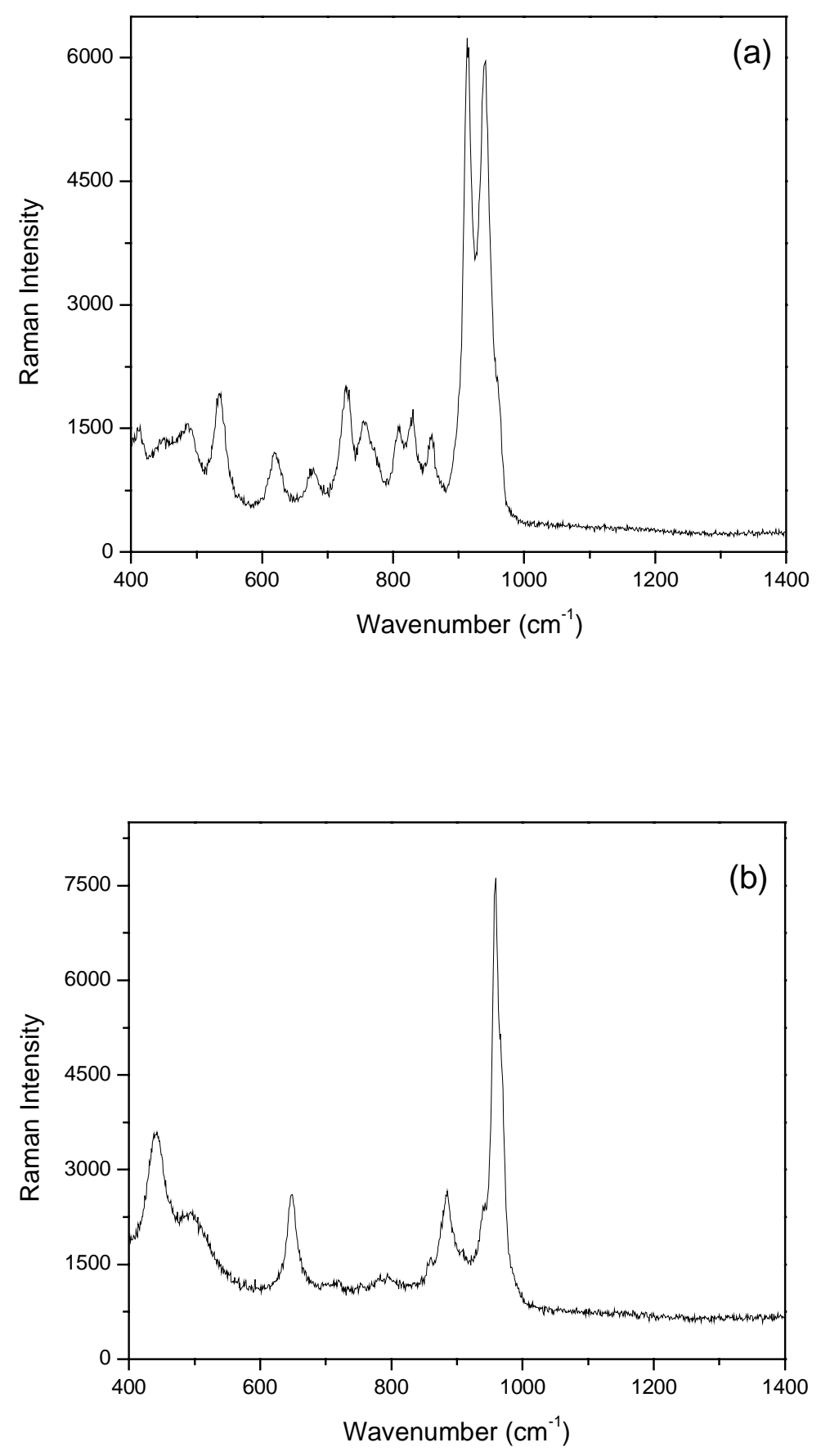

Figure S9. Raman spectra for (a) $\mathrm{Ba}_{2.5}\left(\mathrm{VO}_{2}\right)_{3}\left(\mathrm{SeO}_{3}\right)_{4} \cdot \mathrm{H}_{2} \mathrm{O}$, and (b) $\mathrm{La}\left(\mathrm{VO}_{2}\right)_{3}\left(\mathrm{TeO}_{6}\right) \cdot 3 \mathrm{H}_{2} \mathrm{O}$ 

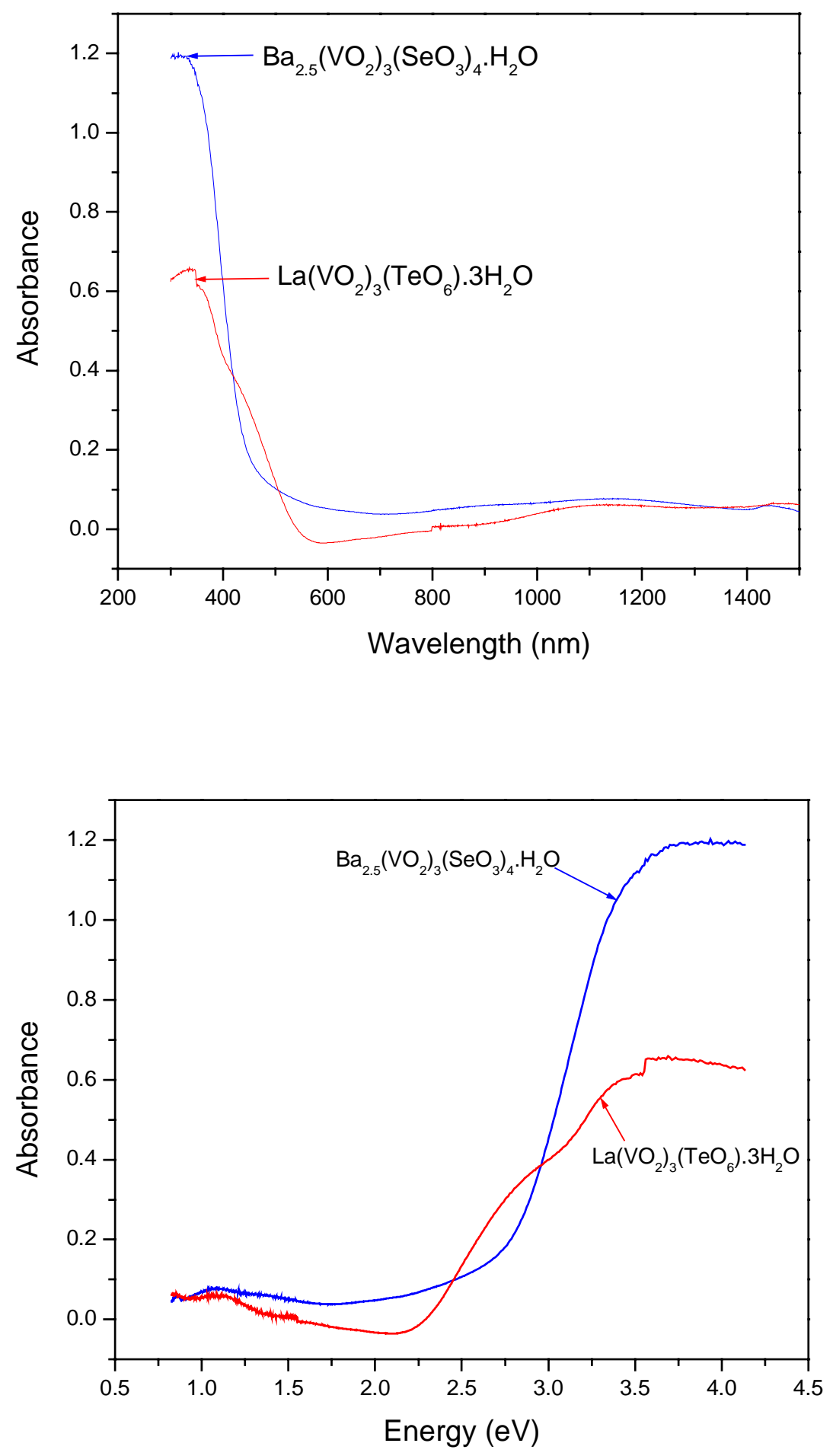

Figure S10. Diffuse reflectance spectra for $\mathrm{Ba}_{2.5}\left(\mathrm{VO}_{2}\right)_{3}\left(\mathrm{SeO}_{3}\right)_{4} \cdot \mathrm{H}_{2} \mathrm{O}$, and $\mathrm{La}\left(\mathrm{VO}_{2}\right)_{3}\left(\mathrm{TeO}_{6}\right) \cdot 3 \mathrm{H}_{2} \mathrm{O}$ 


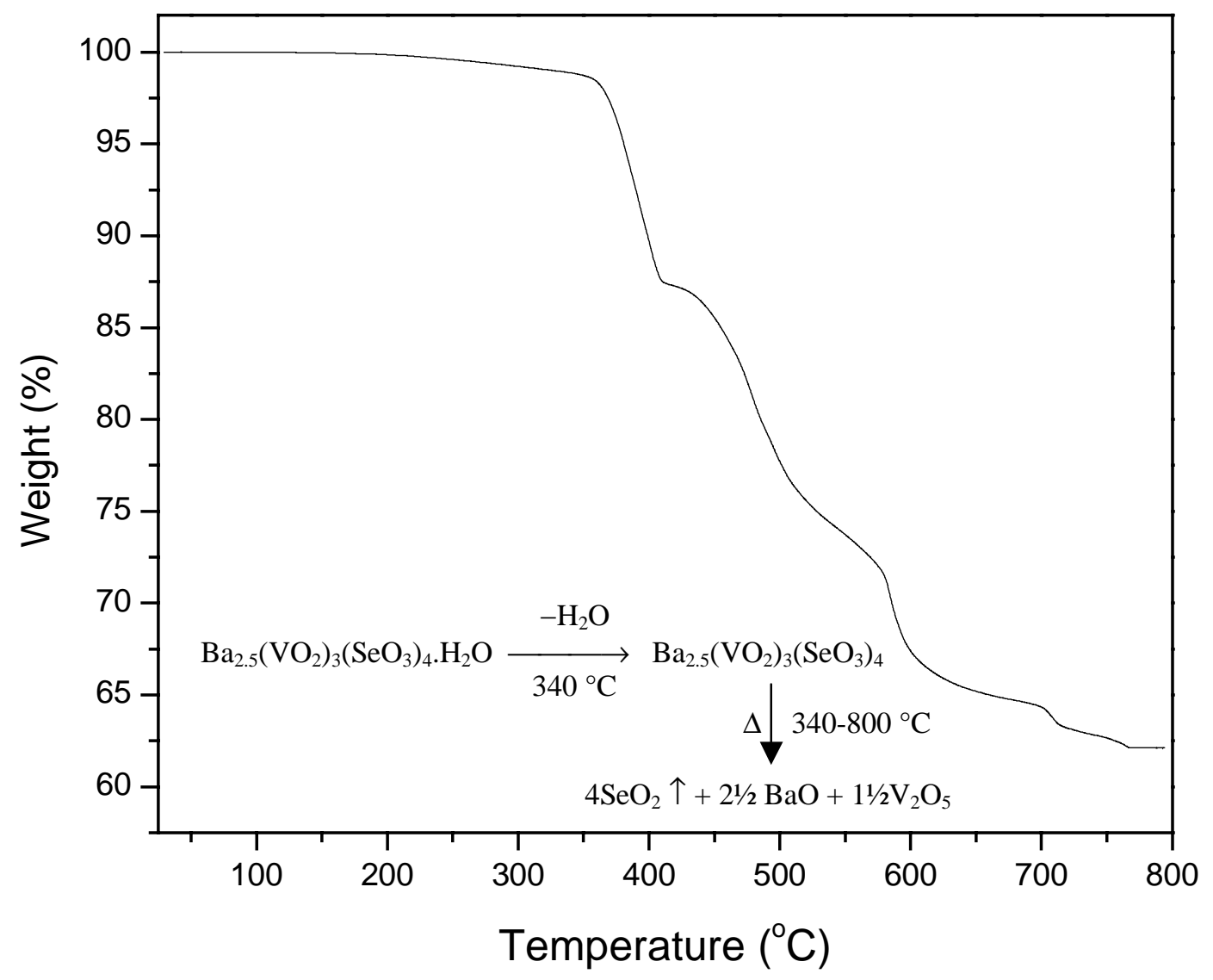

Figure S11. Thermogravimetric analysis curve for $\mathrm{Ba}_{2.5}\left(\mathrm{VO}_{2}\right)_{3}\left(\mathrm{SeO}_{3}\right)_{4} \cdot \mathrm{H}_{2} \mathrm{O}$ 


\section{Isotherm}
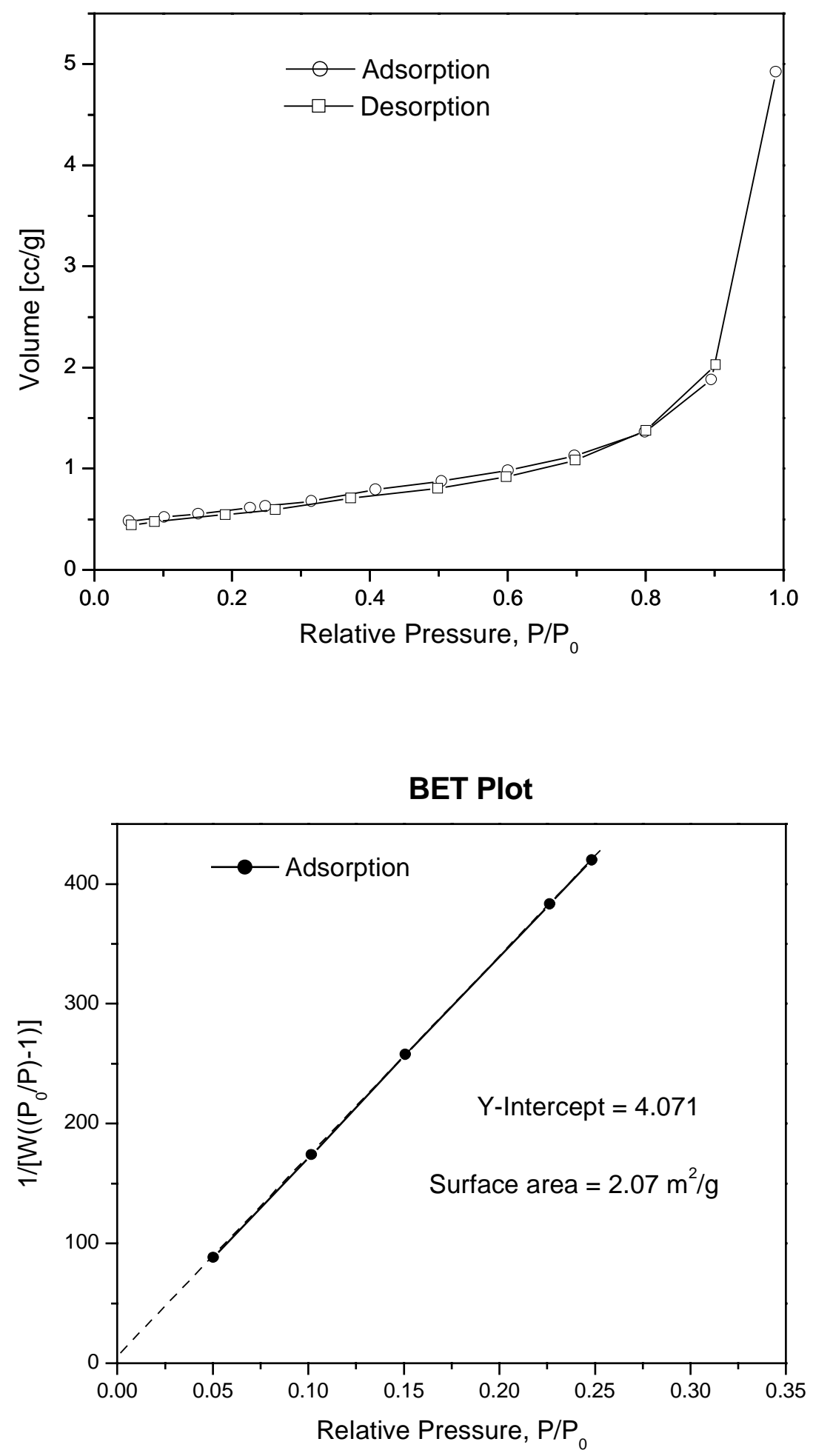

Figure S12. Nitrogen Adsorption Isotherm and BET plot for $\mathrm{Ba}_{2.5}\left(\mathrm{VO}_{2}\right)_{3}\left(\mathrm{SeO}_{3}\right)_{4}$. 


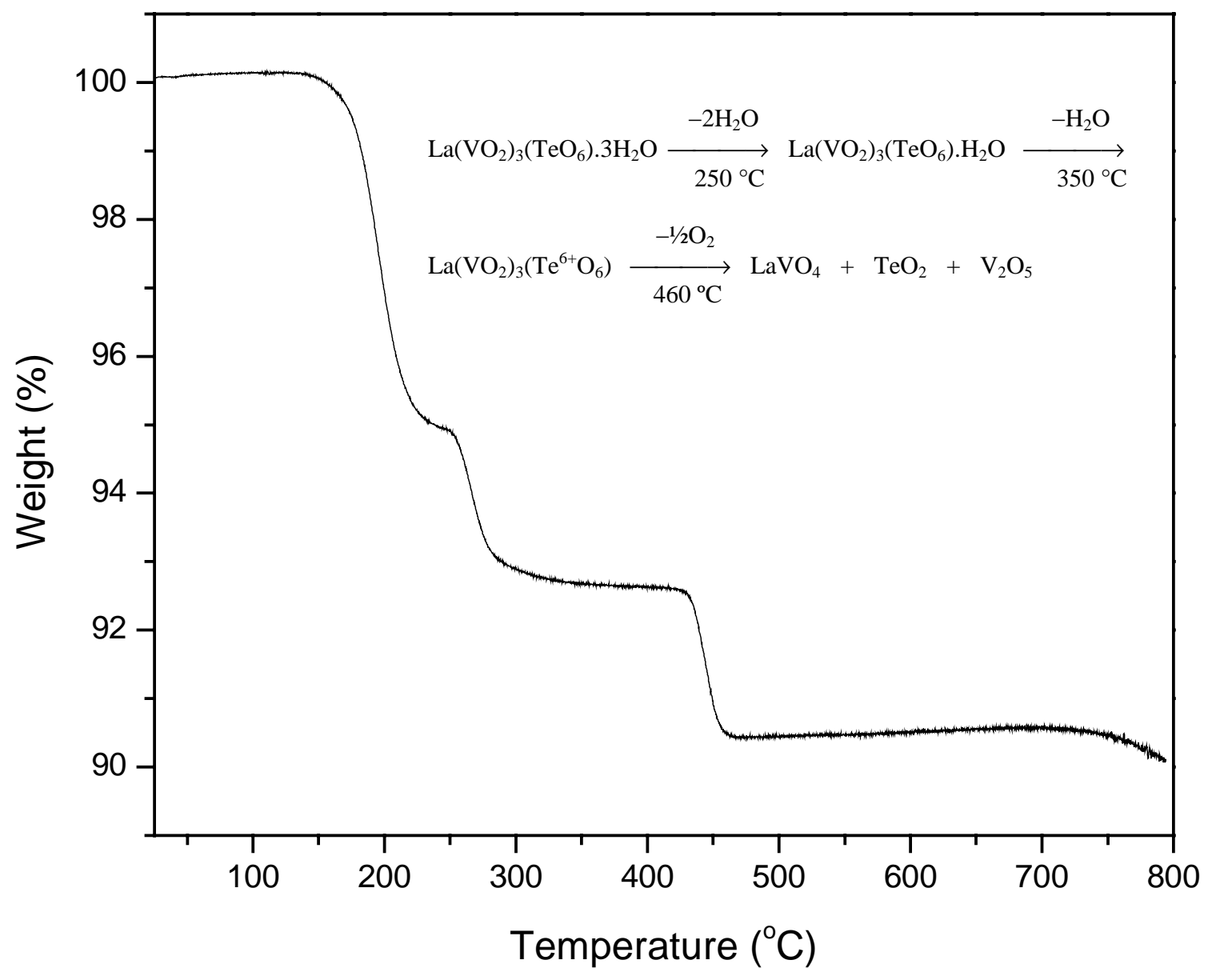

Figure S13. Thermogravimetric analysis curve for $\mathrm{La}\left(\mathrm{VO}_{2}\right)_{3}\left(\mathrm{TeO}_{6}\right) \cdot 3 \mathrm{H}_{2} \mathrm{O}$ 